\title{
Wetting and capillary nematization of a hard-rod fluid: A simulation study
}

\author{
Marjolein Dijkstra, ${ }^{1}$ René van Roij, ${ }^{2}$ and Robert Evans ${ }^{3}$ \\ ${ }^{1}$ Debye Institute, Soft Condensed Matter Physics, Utrecht University, Princetonplein 5, 3584 CC Utrecht, The Netherlands \\ ${ }^{2}$ Institute for Theoretical Physics, Utrecht University, Princetonplein 5, 3584 CC Utrecht, The Netherlands \\ ${ }^{3}$ H.H. Wills Physics Laboratory, University of Bristol, Tyndall Avenue, Bristol BS8 1TL, United Kingdom
}

(Received 27 November 2000; published 18 April 2001)

\begin{abstract}
We present results of a simulation study of a fluid of hard spherocylinders with a length-to-diameter ratio of 15 in contact with a planar hard wall and confined by two parallel hard walls. A Monte Carlo method is developed for simulating fluids in contact with a single wall. Using this method, we find a transition from a uniaxial to a biaxial surface phase, followed, at larger bulk densities, by the formation of a thick nematic film, with the director parallel to the wall, at the wall-isotropic fluid interface. As the density far from the wall $c_{b}$ approaches the value at bulk isotropic-nematic coexistence $c_{I}$, the thickness of the nematic film appears to increase as $-\ln \left(c_{I}-c_{b}\right)$. For a fluid confined by two parallel hard walls, a first-order capillary nematization transition is found. The phase equilibria are determined by Gibbs ensemble Monte Carlo simulations for several wall separations. The difference in the coexisting densities of the capillary condensed nematic and isotropic phases becomes smaller upon decreasing the wall separation, and no capillary nematization transition is found when the wall separation is smaller than about twice the length of the spherocylinders. These features imply that the capillary nematization transition ends in a capillary critical point at a critical wall separation. Our simulation results are fully consistent with the findings of our recent theoretical study of the Zwanzig model for a hard-rod fluid.
\end{abstract}

DOI: 10.1103/PhysRevE.63.051703

PACS number(s): 64.70.Md, 64.60.Cn, 68.08.Bc

\section{INTRODUCTION}

Although liquid crystals have been known since 1888, they were initially viewed as a scientific curiosity [1]. However, during the past few decades, many important industrial applications of liquid crystals have been found arising from the special light scattering and dielectric properties of these substances, and the fabrication of liquid crystalline displays has developed into a multi-billion-dollar industry. Much experimental and theoretical effort has been spent on understanding the phase behavior of liquid crystals. The statistical mechanical theory of liquid crystals began with the seminal work of Onsager in the early 1940s. He was inspired by the experimental observations of a transition from an isotropic $(I)$ to an orientationally ordered nematic $(N)$ phase in colloidal systems of vanadiumpentoxide $\mathrm{V}_{2} \mathrm{O}_{5}$ [2] and of tobacco mosaic virus (TMV) particles [3]. Onsager modeled these systems as a fluid of hard rods and showed that an entropydriven isotropic-nematic transition occurs when the density is sufficiently large [4]. Onsager's theory becomes exact when the length-to-diameter ratio of the rods goes to infinity. For hard rods (spherocylinders) with a finite length-todiameter ratio, the bulk phase behavior has been determined by computer simulations $[5,6]$ and by density-functional theory [7]; good agreement has been found between the results of the two approaches. More important for practical applications is obtaining a fundamental understanding of surface effects in liquid crystals. There are several very basic questions that have not been answered in a fully convincing fashion. For instance, what happens to a fluid of rodlike molecules in contact with a single hard wall or confined by two hard walls? Several predictions have been made by densityfunctional-theory calculations. However, such calculations are difficult as the wall may break the uniaxial symmetry of the one-particle distribution function giving rise to a biaxial profile. Thus approximations have often been made for the one-particle distribution function. Poniewierski and Holyst used a simple local approximation for the one-particle distribution function in their study of a fluid of hard rods in contact with a single planar hard wall [8]. Their approximation ignores the possibility of biaxiality, which was remedied in a more recent paper by Poniewierski, who studied the stability of the uniaxial density profiles with respect to biaxial ones using a bifurcation analysis [9]. Poniewierski found that the onset of biaxiality occurs at a bulk density that is $15 \%$ below that of the bulk $I-N$ transition, but did not calculate biaxial profiles. Nor did he address the issue of complete wetting by nematic of the hard-wall isotropic interface. A full densityfunctional calculation of biaxial density profiles has been performed by Chen and Cui for a fluid of hard-core semiflexible polymers near a hard wall [10]. They find a very weakly first-order uniaxial to biaxial transition at a bulk density well below that of the bulk $I-N$ transition. They also observe the formation of a (biaxial) nematic film with the director parallel to the wall at the wall-isotropic fluid interface. The thickness of this film appears to diverge as bulk $I-N$ coexistence is approached. This is a strong indication of complete wetting.

For the case of a hard-rod fluid confined by two parallel hard walls, we are only aware of the study of Mao et al. [11], where they employed the Onsager functional under the assumption that the density profiles are uniaxial for state points far below the bulk $I-N$ transition. They compared these results with those of computer simulations. The aim of this work is to calculate the depletion (or solvation) force between the plates due to confinement of the rods. Another related theoretical and simulation study by Allen is concerned with hard ellipsoids in external anchoring fields 
$[12,13]$. The focus of Ref. [12] is on elasticity and anchoring of liquid crystals and that of Ref. [13] is on the structure of the IN interface. References [11-13] are not concerned with the phase behavior of the confined fluid, which is the topic of the present paper.

Here, we study a fluid of hard spherocylinders with a length-to-diameter ratio of 15 in contact with a planar hard wall and confined by two parallel hard walls. We develop a Monte Carlo method for simulating a fluid in contact with a single wall in order to study adsorption and wetting phenomena. This method is described in Sec. II, and in Sec. III we apply it to a fluid of hard spherocylinders. We find a transition from a uniaxial to biaxial phase at densities well below that of the bulk $I-N$ transition, followed by the formation of a nematic film with the director parallel to the wall whose thickness appears to diverge as bulk $I-N$ coexistence is approached. By performing Gibbs ensemble Monte Carlo simulations of the fluid of spherocylinders between two parallel hard walls, we find a first-order capillary nematization transition and determine the coexistence densities for several wall separations; these results are discussed in Sec. IV. We conclude in Sec. V by summarizing the results and making comparisons with those obtained from the Zwanzig model $[14,21]$. A preliminary account of some of the simulation results reported here can be found in Ref. [14].

\section{A SIMULATION METHOD FOR A FLUID IN CONTACT WITH A SINGLE WALL}

In order to investigate the adsorption and, in particular, the wetting properties of a fluid, it is important to study a bulk fluid in contact with a single wall. This single-wall system is difficult to treat in computer simulations, since the fluid in contact with the wall at $z=0$ cannot be treated with periodic boundary conditions in the $z$ direction. Consequently, in many computer simulations of adsorption phenomena, the fluid is confined between two identical walls in the $x y$ plane, located at $z=0$ and $z=L_{z}$. The additional wall at $z=L_{z}$ should not affect the adsorption of the fluid at the wall of interest at $z=0$ provided $L_{z}$ is large enough that there is a flat portion of the density profile in the central region of the slit. A drawback of using large $L_{z}$ is, however, that long simulation times are needed, which may hamper a systematic study of the growth of a thick wetting film. Using smaller values of $L_{z}$ can lead to finite-size (capillary) effects, and these may hinder the observation of the single-wall phenomena of interest. Such effects are especially important in the grand-canonical ensemble where capillary condensation (or evaporation) occurs as the chemical potential approaches its value at bulk coexistence and the phase with the wetting films at the walls becomes metastable with respect to condensation [15]. Observation of the growth of such films then becomes problematic.

In this paper, we develop a simulation method for treating a fluid in contact with a single wall. We emphasize that our method is not restricted to the particular model investigated here, but can be applied to any fluid and any wall; results for the adsorption and wetting behavior of a hard-sphere fluid at a planar hard wall and for density profiles of a colloid- polymer mixture in contact with a planar hard wall will be presented elsewhere [16]. The simulation method we present is similar in spirit to performing iterative solutions of the Euler-Lagrange equations for the density profiles of fluidwall systems given by density-functional theory (DFT) [17]. The Euler-Lagrange equations are solved iteratively on a grid under the condition that the density far from the wall reaches a bulk density $\rho_{b}$, which corresponds to the imposed chemical potential. In our simulation method, we impose a self-consistently determined number density $\rho_{b}$ far from the wall using a penalty function that suppresses large deviations from $\rho_{b}$.

Consider a fluid of $N$ particles in a box with lateral dimensions $L_{x}, L_{y}$, and $L_{z}$. The wall of interest, in our particular case a planar hard wall, is positioned at $z=0$. As discussed above, one often confines the fluid between two identical walls, but then capillary effects can occur and may mask or compete with the adsorption phenomena of interest. An alternative procedure to simulate such a system would be to impose periodic boundary conditions in the $x$ and $y$ directions, but not in the $z$ direction. Clearly, one must limit the positions of the particles such that the $z$ coordinate of particle $i$, say $z_{i}$, cannot become larger than some value $L_{z}$, i.e., $z_{i}$ $\leqslant L_{z}$. Such a system corresponds to a fluid confined between the wall of interest, located at $z=0$, and a different wall located at $z=L_{z}$. Such a procedure was employed by Finn and Monson [18] in their simulation study of prewetting for a Lennard-Jones gas at a (weakly) attractive wall. They chose the second wall to be hard so that no wetting occurred at this wall. Clearly, one must choose the second wall appropriate to the physical system under investigation. For hard rods, it is not obvious what type of wall potential would ensure a flat density profile near the second wall. Any substantial increase in the local density might result in surface transitions occurring in the layers adsorbed at the second wall, and these should be avoided. In his study of surface anchoring in nematic phases of hard ellipsoids, Allen [12] imposed an external orienting field in a region adjoining his second wall leading to the formation of a highly oriented "ffilm" with a higher degree of nematic order (and a higher contact density) than in the bulklike region in the center of the slit [19]. Problems of smooth matching to bulk are circumvented in the present procedure where an additional external potential or penalty function $V(z)$, designed to suppress density deviations from a self-consistently determined density $\rho_{b}$, is imposed in a region close to the second wall at $z=L_{z} . V(z)$ acts to suppress any surface transition at the second wall. To this end, we calculate the density profile, with data averaged over bins of width $\delta z$, using the following external potential for bin $j$ :

$$
\beta V\left(z_{j}\right)= \begin{cases}\frac{1}{2} k\left(\frac{n_{j}}{L_{x} L_{y} \delta z}-\rho_{b}\right)^{2}, & z_{\text {imp }} \leqslant z_{j} \leqslant L_{z} \\ 0 & \text { otherwise }\end{cases}
$$

where $\beta=\left(k_{B} T\right)^{-1}, k>0$ determines the magnitude of the allowed density fluctuations, $n_{j}$ is the number of particles present in bin $j$, and $\rho_{b}$ is the self-consistently determined 
density far from the wall of interest, corresponding to the bulk density. The external potential or penalty function applies only in bins $j$ with $z_{\text {imp }} \leqslant z_{j} \leqslant L_{z}$, where $0 \leqslant z_{\text {imp }} \leqslant L_{z}$.

It is important to note that in density-functional theory, the density far away from the wall is imposed by the fixed chemical potential. In principle, our simulation technique can be performed in the grand-canonical ensemble. However, for dense hard-core fluids, it is almost always impossible to insert particles, leading to poor sampling of phase space and poor statistics. Thus we have chosen to keep the number of particles fixed. Our procedure is as follows.

(i) We fix the number of particles $N$, the dimensions of the simulation box $L_{x}, L_{y}$, and $L_{z}$, and we estimate the density far from the wall of interest, denoting this as $\rho_{b}$.

(ii) We perform a simulation with this initial estimate for $\rho_{b}$ and calculate the density profile. The average density $\rho_{\mathrm{av}}$ far from the wall of interest is obtained by averaging over the bins, which are sufficiently far from the wall and which show a sufficiently flat profile.

(iii) We repeat step (ii) using $\rho_{\mathrm{av}}$ for $\rho_{b}$ until the new $\rho_{\mathrm{av}}$ equals the previous value within some given tolerance.

We conclude this section with some remarks. (i) This simulation technique can be used for any wall-particle potential and any particle-particle interaction and can easily be extended to fluid mixtures. (ii) The simulations can also be performed in the grand-canonical ensemble for systems where insertion of particles is not problematic. (iii) We emphasize that in order to obtain a flat density profile far from the wall of interest, it is important that $L_{z}$ is sufficiently large. In the next section, we apply this method to a fluid of hard spherocylinders in contact with a hard wall.

\section{A HARD-SPHEROCYLINDER FLUID IN CONTACT WITH A SINGLE HARD WALL}

We perform Monte Carlo simulations of a fluid of hard spherocylinders with a length-to-diameter ratio of $L / D=15$. This model exhibits a first-order transition from an isotropic to a nematic phase in bulk and we first determine the bulk IN coexistence by standard Gibbs ensemble simulations [20] of 2300 particles. These yield coexistence densities $c_{I}=3.675$ \pm 0.003 and $c_{N}=4.300 \pm 0.003$, where we define the dimensionless density $c=(L+D)^{2} D \rho$ with $\rho$ the number density. We then perform a simulation study of the isotropic bulk fluid in contact with a single hard wall using the method described in Sec. II. The transverse dimensions of the simulation box are $L_{x} / D=L_{y} / D=36.74$ and the second wall is at $L_{z} / D=64$ or $L_{z} / D=112$. The number of particles is chosen such that we obtain an isotropic fluid far from the first wall, i.e., the dimensionless density far from the hard wall $c_{b}$ $=(L+D)^{2} D \rho_{b}<c_{I}$. This corresponds to about 400-3000 particles for our choices of $c_{b}$. We applied the additional external potential Eq. (1) with a value of $z_{\text {imp }}$ such that $\mid L_{z}$ $-z_{\text {imp }} \mid=0.5 L$ and chose $k D^{6}$ in the range $\left(2 \times 10^{3}\right)-(2$ $\left.\times 10^{6}\right)$. For hard particles, temperature $T$ is not a significant quantity and we set $k_{B} T=\beta^{-1}=1$ for all calculations.

We measured the density profile and the eigenvalues of the standard $3 \times 3$ nematic order-parameter tensor

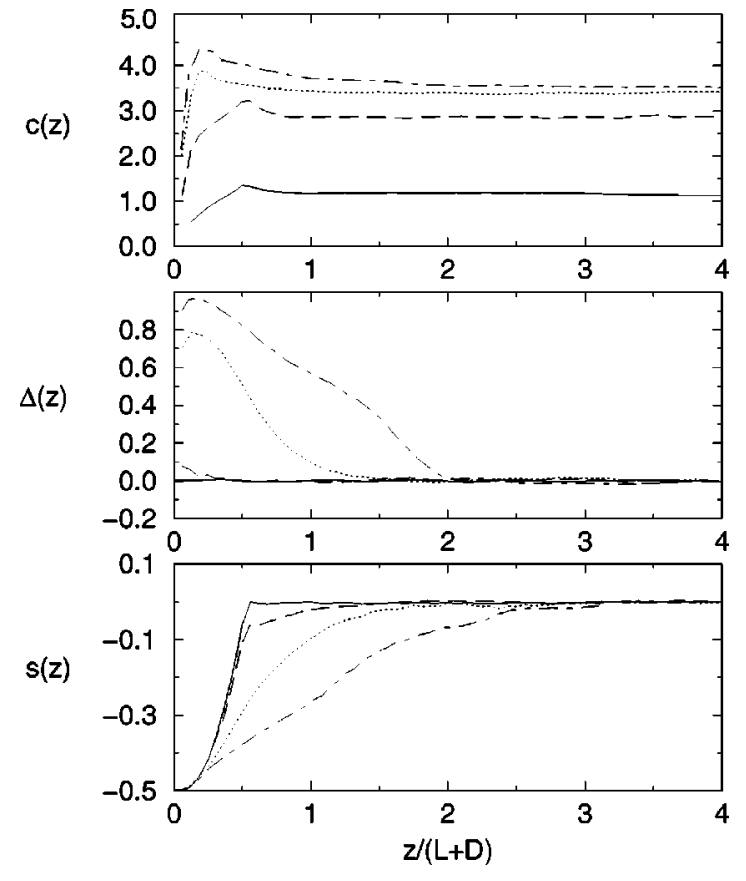

FIG. 1. Density and order-parameter profiles of a hardspherocylinder fluid in contact with a single hard wall located at $z$ $=0$. The dimensionless bulk density $c_{b}=(L+D)^{2} D \rho_{b}$ far from the wall is $c_{b}=1.169$ (solid line), $c_{b}=2.855$ (dashed line), $c_{b}=3.379$ (dotted line), and $c_{b}=3.515$ (dashed-dotted line). The total density is $c(z)$, the nematic order parameter is $s(z)$, and the biaxial order parameter is $\Delta(z)$.

$$
Q_{\alpha \beta}\left(z_{j}\right)=\left\langle\frac{1}{n_{j}} \sum_{i=1}^{n_{j}}\left(\frac{3}{2} u_{\alpha}^{i} u_{\beta}^{i}-\frac{\delta_{\alpha \beta}}{2}\right)\right\rangle, \quad \alpha, \beta=x, y, z,
$$

where $u_{\alpha}^{i}$ is the $\alpha$ component of the unit orientation vector of particle $i, n_{j}$ is the number of particles present in bin $j$, and $\delta_{\alpha \beta}$ is the Kronecker delta. Diagonalizing this tensor gives three orientational ordering eigenvalues $\lambda_{+}, \lambda_{0}$, and $\lambda_{-}$for each bin. We analyze our results by considering two independent order-parameter profiles: (i) the nematic orderparameter profile is given by the lowest eigenvalues $s(z)$ $=\lambda_{-}(z)$, and (ii) the biaxial order-parameter profile $\Delta(z)$ $=2\left[\lambda_{+}(z)-\lambda_{0}(z)\right] / 3$. More than $5 \times 10^{4}$ Monte Carlo sweeps were allowed for equilibration and the profiles in bins of width $\delta z=0.0625(L+D)$ were accumulated over a further $5 \times 10^{6}$ sweeps (one sweep is one attempted move per particle). The density and order-parameter profiles are shown in Fig. 1 for four different values of $c_{b} \leqslant 3.535$. We discuss first the results for $c_{b}=1.169$ (solid lines). Figure 1 shows that the density profile $c(z)$ is at a minimum at $z=0$, indicating a depletion of particles close to the wall. $c(z)$ increases with $z$ and reaches a maximum at $z=L / 2$, where there is a kink in $c(z)$. This is in agreement with the DFT results of Ref. [9]. For higher $z, c(z)$ decreases and reaches the bulk value at $z \simeq L$. We observe that using our simulation method, the density profile remains flat at large $z$. Turning to the nematic order-parameter profile $s(z)$ in Fig. 1, we find that $s(z=0)=-0.5$ at the wall and increases to 0 for larger 
$z$. $s(z)$ also shows a kink at $z=L / 2$. The biaxial orderparameter profile $\Delta(z)=0$ for all $z$. Thus, we find a uniaxial distribution close to the wall as $s(z)<0$ and $\Delta(z)=0$, and an isotropic distribution for $z>L$ as $s(z)=0$ and $\Delta(z)=0$. For $c_{b}=2.855$ (dashed lines), most of the features are similar to those for $c_{b}=1.169$. However, we now find nonzero values for $\Delta(z)$ close to the wall, corresponding to a biaxial distribution of the rods. In order to locate the uniaxial-biaxial (UB) transition, we perform a few shorter runs for 1.169 $<c_{b}<2.855$. We estimate that the onset of biaxial ordering $\Delta(z) \neq 0$ first occurs at about $c_{\mathrm{UB}}=2.80 \pm 0.05$ or $c_{\mathrm{UB}} / c_{I}$ $=0.76$, in qualitative agreement with the theoretical prediction for the Zwanzig model $\left(c_{\mathrm{UB}} / c_{I} \simeq 0.819\right)[14,21]$, in which the orientations of the particles are restricted to three orthogonal directions, and with the density-functional-theory results for freely rotating infinitely elongated spherocylinders $\left(c_{\mathrm{UB}} / c_{I} \simeq 0.847\right)$ [9]. Thus, for $c_{b}<c_{\mathrm{UB}}$ we find uniaxial symmetry for all $z$, whereas biaxiality sets in when $c_{b}$ $>c_{\mathrm{UB}}$. We did not attempt to ascertain the nature of the UB transition. Recall that within our mean-field treatment for the Zwanzig model, there is compelling evidence that the transition is second-order [14,21] but the Zwanzig model has restricted orientations. Understanding the precise nature of the UB transition in freely rotating hard-rod systems where soft modes are present remains a challenge for simulation and theory.

If we increase $c_{b}$ beyond $c_{\mathrm{UB}}$ to $c_{b}=3.379$ and 3.515 (dotted and dashed-dotted lines), we find that the kink at $z$ $=L / 2$ disappears in $c(z)$ and $s(z)$. We still find a maximum in $c(z)$ but shifted to smaller $z . \Delta(z)$ increases its value close to the wall when $c_{b}$ is increased, corresponding to a stronger biaxial ordering close to the wall. We also find that the $z$ interval where $\Delta(z)$ and $s(z)$ are nonzero increases for increasing $c_{b}$, signifying that the width of the nematic film (with the director parallel to the wall) is increasing with $c_{b}$. This can be better appreciated in Fig. 2, which shows the density and order-parameter profiles for $c_{b}=3.535,3.633$, and 3.663, i.e., closer to the bulk transition at $c_{I}=3.675$. We clearly observe in the density and in both order-parameter profiles that the film with in-plane nematic ordering has increasing thickness for increasing $c_{b}$. The simulations lend strong support for complete orientational wetting in the limit $c_{b} \rightarrow c_{I}$. It is worth noting that for the highest value of $c_{b}$, the nematic film occupies a very large fraction of the simulation box and we do begin to run out of "bulk." The results for this state point might possess substantial error bars. This issue will be discussed in more detail in Sec. V.

More quantitative support for the complete orientational wetting scenario is provided by the measured logarithmic increase of the adsorption $\Gamma$ as a function of $\left(c_{I}-c_{b}\right) / c_{I}$, where $\Gamma$ is defined as

$$
\Gamma=\frac{1}{L} \int_{0}^{L_{z}} d z\left[c(z)-c_{b}\right]
$$

Figure 3 indicates that $\Gamma$ increases logarithmically as $c_{b}$ $\rightarrow c_{I}$. The adsorption can be fitted by $\Gamma=A_{1}+A_{2} \ln \left[\left(c_{I}\right.\right.$ $\left.\left.-c_{b}\right) / c_{I}\right]$, with fit parameters $A_{1}=-2.58458$ and $A_{2}=$

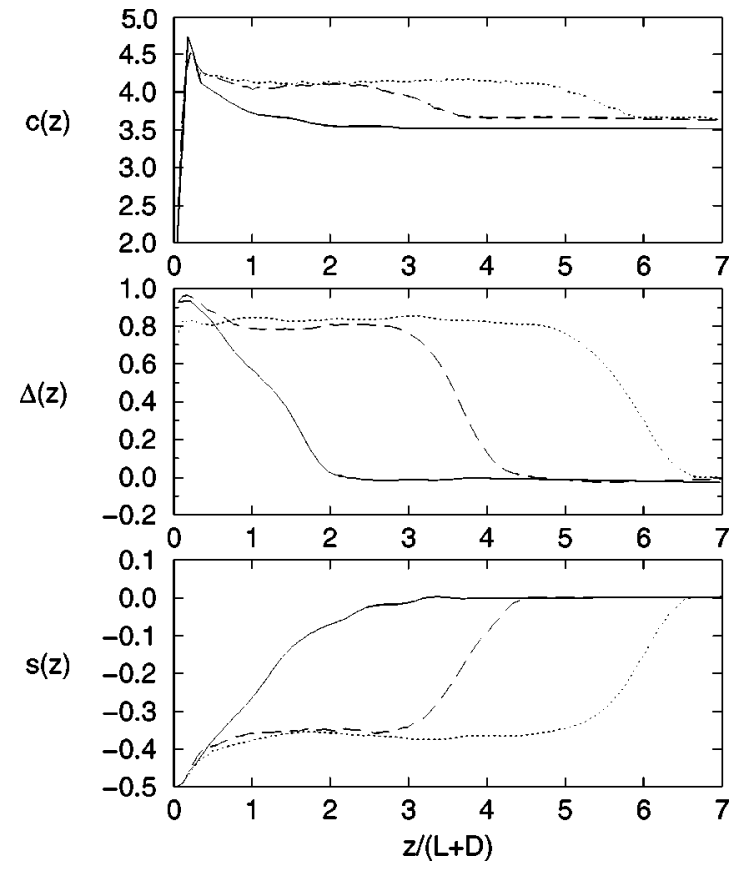

FIG. 2. Same as in Fig. 1, but with $c_{b}=3.535$ (solid line), $c_{b}$ $=3.633$ (dashed line), and $c_{b}=3.663$ (dotted line). Note that the bulk isotropic-nematic transition occurs at density $c_{I}=3.675$.

-0.9138972 for $c_{b}>3.515$. For comparison, we plot the result of this fit as the solid line in Fig. 3. In principle, the complete wetting scenario could be confirmed by calculating the contact angle, defined by [21]

$$
\cos \theta=\frac{\gamma_{\mathrm{WI}}-\gamma_{\mathrm{WN}}}{\gamma_{\mathrm{IN}}}
$$

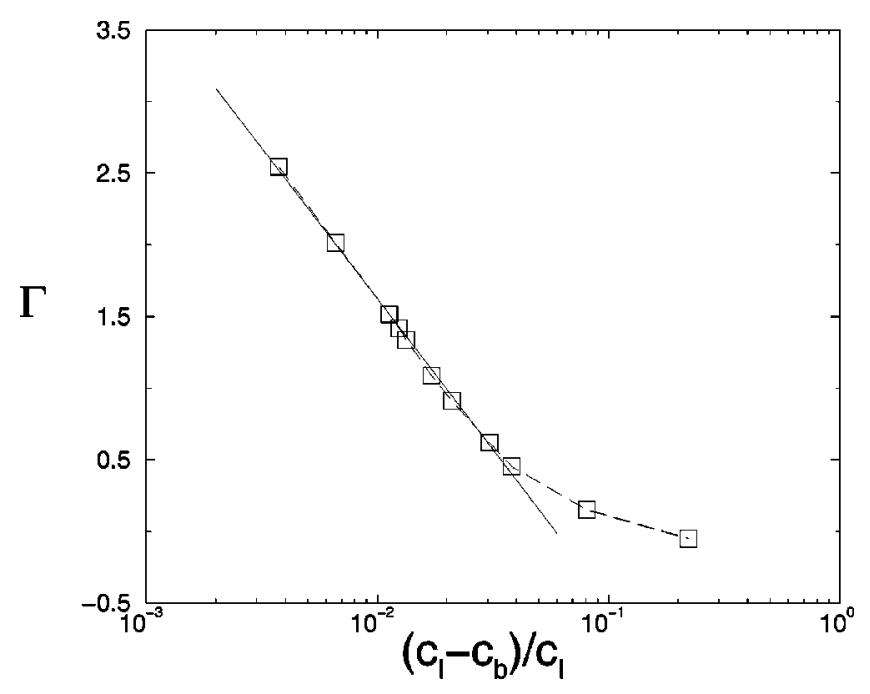

FIG. 3. Adsorption $\Gamma$ of a fluid of hard spherocylinders in contact with a single hard wall as a function of the difference between the bulk isotropic-nematic coexistence density $c_{I}$ and the density far from the wall $c_{b}$. The simulation results are denoted by squares joined by the dashed line to guide the eye. The adsorption can be fit by $\Gamma=A_{1}+A_{2} \ln \left[\left(c_{I}-c_{b}\right) / c_{I}\right]$, with fit parameters $A_{1}=-2.58458$ and $A_{2}=-0.9138972$ for $c_{b}>3.515$, and this fit is denoted by the solid straight line. 

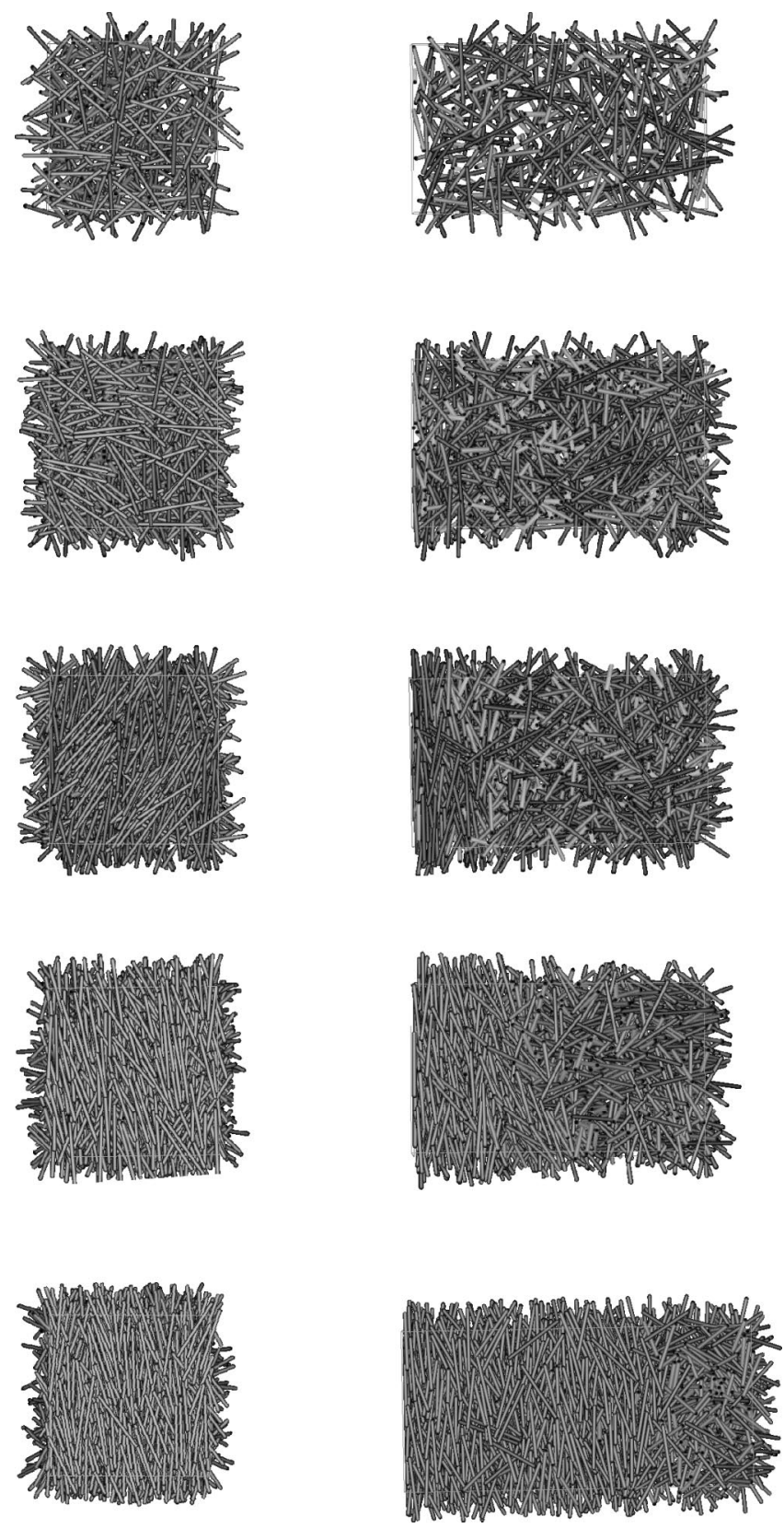

FIG. 4. Snapshots of the hard-spherocylinder fluid for $c_{b}$ $=1.169,2.855,3.379,3.535$, and 3.633 (from top to bottom). Each left-hand-side figure shows a snapshot of the fluid in the $x y$ plane taken from the wall at $z=0$ looking into the fluid, while each righthand side shows the fluid in the $x z$ plane with the hard wall on the left. Note that in the last snapshot, the box length $L_{z} / D=112$, i.e., $L_{z} /(L+D)=7$, whereas the length of the cell shown here is 5 ( $L$ $+D)$.

where $\gamma_{\mathrm{WI}}, \gamma_{\mathrm{WN}}$, and $\gamma_{\mathrm{IN}}$ are, respectively, the interfacial tension between the wall-isotropic fluid phase with $c_{b}=c_{I}$, the wall-nematic phase with $c_{b}=c_{N}$, and the isotropicnematic interface at bulk coexistence. A vanishing contact angle would correspond to complete wetting of the wallisotropic (WI) fluid interface by the nematic phase. However, unlike the corresponding analysis for the Zwanzig model, where it is straightforward to confirm $\theta=0[14,21]$, it is an extremely demanding and almost impossible task to confirm the vanishing contact angle scenario by simulations as one requires all three interfacial tensions with sufficient statistical accuracy. We did not attempt to proceed with this route [22].

More visual information about the ordering at the hard wall is shown in Fig. 4, where we show snapshots for $c_{b}$ $=1.169,2.855,3.379,3.535$, and 3.633, five of the states for which we showed profiles in Figs. 1 and 2. The left-hand side of each figure shows a snapshot of the fluid in the $x y$ plane taken from the hard wall at $z=0$, while the right-hand side shows the fluid in the $x z$ plane. For $c_{b}=1.169$, we clearly observe a uniaxial distribution, while at $c_{b}=2.855$ some small degree of biaxiality has set in. At $c_{b}$ $=3.379,3.535$, and 3.633, we observe that the in-plane nematic film grows steadily, reaching a thickness of about 4( $L$ $+D)$ for $c_{b}=3.633$.

\section{A HARD-SPHEROCYLINDER FLUID CONFINED BY TWO PARALLEL HARD WALLS}

The results of the preceding section show that for $c_{b}$ $\rightarrow c_{I}$, a hard wall favors planar nematic (director parallel to the wall) over isotropic ordering. In surface tension terms, $\gamma_{\mathrm{WI}}>\gamma_{\mathrm{WN}}$ so that $\cos \theta>0$. Simple arguments based on the Kelvin equation (e.g., Ref. [15]) would then imply that for the fluid confined between two hard walls, capillary condensation of the nematic phase should occur prior to the bulk IN transition, i.e., for some value of $c_{b}<c_{I}$, when the wall separation $H$ is finite. Such a scenario is found in calculations based on the Zwanzig model, where $\cos \theta=1$, and one observes a first-order transition from an isotropic phase, with biaxial nematic films at the hard walls, condensing to a nematic phase that fills the slit [14,21]. This transition is termed capillary nematization. We seek the equivalent transition in the present model by performing a simulation study of a fluid of hard spherocylinders between two parallel hard walls. We do indeed find a first-order capillary nematization transition from a phase with biaxial films $(B)$ to a capillary condensed nematic phase $(C)$. The phase equilibria are determined by performing Gibbs ensemble simulations of a fluid of hard spherocylinders with the same length-to-diameter ratio of $L / D=15$, at fixed wall separations $h=H /(L+D)=4,3,2.5$, 2.375, and 2.25. In a Gibbs ensemble simulation for confined systems, the two coexisting phases are simulated in two separate boxes, which can exchange area and particles, while the wall separation is kept constant. The exchange of particles ensures equal chemical potential for both phases, while the exchange of area ensures equal fluid-surface interfacial tension in the same spirit as the exchange of volume ensures equal pressure in bulk systems. A full description of the Gibbs ensemble simulation technique applied to confined systems is given by Panagiotopoulos [23].

The transverse dimensions of the initial simulation boxes are $L_{x} / D=L_{y} / D=36.74$ and the number of particles $N$ varies from 1700 to 2100 particles for the largest wall separation. The coexisting densities were accumulated over 5 $\times 10^{5}$ sweeps, where one sweep consists of one attempted move per particle, one attempted area change, and one attempted particle swap between the two boxes. 


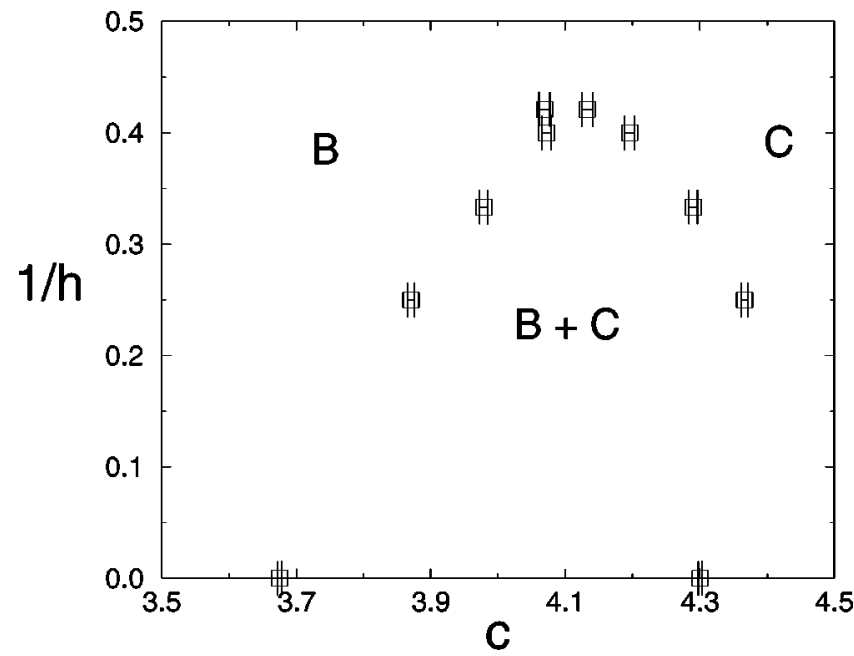

FIG. 5. The coexistence curve for the isotropic phase with biaxial films $(B)$ and capillary condensed nematic phase $(C)$ transition as a function of the dimensionless number density $c$ in the slit and the dimensionless inverse wall separation $1 / h$. The symbols are the results of Gibbs ensemble Monte Carlo simulations for the confined fluid. There is no capillary nematization for $h \leqslant 2.25$. Note that the two points on the $h=\infty$ axis are the bulk coexistence values $c_{I}$ and $c_{N}$ obtained from bulk Gibbs ensemble simulations.

The resulting phase diagram is shown in Fig. 5 in terms of the inverse wall separation $h^{-1}$ and the dimensionless number density $c=N(L+D)^{2} D /\left(H L_{x} L_{y}\right)$ in the slit. We observe coexistence between phase $(B)$ and a capillary condensed nematic phase $(C)$ provided $h \geqslant 2.375$, whereas no coexistence could be found for $h \leqslant 2.25$. The difference between the coexisting densities decreases smoothly as $h$ is reduced. For $h \leqslant 2.25$, no capillary nematization is found and the slit "fills" continuously as $c_{b}$ is increased towards $c_{I}$. These features are in agreement with the results of the Zwanzig model $[14,21]$ and imply that the capillary nematization transition ends in a capillary critical point at a critical wall separation $h_{c} \simeq 2.3$. This value is quite close to the corresponding result from the Zwanzig model $h_{c} \equiv H_{c} / L=2.08 \pm 0.01$ $[14,21]$. Note also that the shape of the coexistence curve is reminiscent of that obtained from the Zwanzig model. The condensed nematic branch has a positive slope at small values of $1 / h$. This reflects the choice of order parameter $c$ and can be accounted for using estimates based on the Kelvin equation, valid as $1 / h \rightarrow 0$ [21]. Within the Zwanzig model, the biaxial branch acquires a large gradient in the approach to the critical point (although the criticality is standard meanfield-like in the density-functional treatment of Ref. [21]). There is some evidence for a similar feature in the present results, which, once again, could reflect the choice of order parameter, but our simulation data are not sufficiently comprehensive to confirm this. Neither are we in a position to determine the nature of the criticality, i.e., the critical exponents. We conjecture that, as in the case of simple fluids, the criticality of the capillary nematization transition should lie in the two-dimensional Ising universality class since the order parameter (the difference in the average densities at coexistence) is a scalar and correlations may only diverge in the $x-y$ plane. This would imply a flat-topped coexistence curve (exponent $\beta=1 / 8$ ). That we do not observe such a shape may reflect the particular choice of order parameter or that we are insufficiently close to the critical point.

\section{SUMMARY AND DISCUSSION}

We studied a fluid of hard spherocylinders with $L / D$ $=15$ in contact with a planar hard wall and confined by two parallel hard walls. Using a Monte Carlo method developed specifically for simulating a fluid in contact with a single wall, we found a surface transition from a uniaxial to a biaxial phase, followed, at larger bulk densities, by the formation of a thick in-plane nematic film, whose thickness appears to increase as $-\ln \left(c_{I}-c_{b}\right)$, indicating complete wetting of the wall-isotropic fluid interface by the in-plane oriented nematic film. While we believe that the method we have introduced is particularly well-suited for investigating wetting films, we emphasize, once again, that it remains difficult within computer simulation to demonstrate unequivocally complete wetting; one cannot investigate the strict limit of box length $L_{z} \rightarrow \infty$. In our present calculations, we are confident that for $\left(c_{I}-c_{b}\right) / c_{I}>0.01$, corresponding to the profiles in Fig. 1, the two thinner films in Fig. 2, and all the snapshots in Fig. 4, we have sufficient "bulk" fluid far from the hard wall to ensure reliable averaging. We confirmed that the same profiles resulted for $L_{z} / D=64$ and 112. For smaller values of $\left(c_{I}-c_{b}\right) / c_{I}$, the nematic film occupies a very large fraction of the simulation box and we do begin to run out of "bulk." Thus the results for the profiles corresponding to $c_{b}=3.663$ (dotted line) in Fig. 2 and the two highest values of the adsorption $\Gamma$ shown in Fig. 3 might possess substantial error bars. This observation becomes relevant when we make comparison with the corresponding results from the Zwanzig model, where the adsorption diverges logarithmically but with a much smaller prefactor, i.e., the coefficient $\left|A_{2}\right|$ defined below Eq. (3) is 0.235 [21] rather than 0.914, the present value. We should recall that general arguments for complete wetting for fluids with short-ranged forces (at the mean-field level) imply that the wetting film thickness $l$ should diverge as $-\xi_{b} \ln \left[\left(c_{I}-c_{b}\right) / c_{b}\right]$, where $\xi_{b}$ is the bulk correlation length of the phase, which is wetting. For very thick films, $\Gamma \simeq l\left(c_{N}-c_{I}\right) / L$ so that $\left|A_{2}\right|=\xi_{b}\left(c_{N}-c_{I}\right) / L$. Given that $c_{N}-c_{I}$ does not differ greatly between the simulations and the Zwanzig model, our results imply that $\xi_{b} / L$ should differ by about a factor of 4 . This is surprising. It is possible that capillary-wave-like fluctuations in the wetting film act to augment the prefactor, but it is more likely that we have not entered the true asymptotic regime in the simulations. Such issues become important if one attempts to investigate the depinning nematic-isotropic interface within the present simulation scheme. Provided the in-plane nematic does wet completely the hard-wall isotropic interface, the nematic-isotropic interface will depin continuously from the wall as $c_{b} \rightarrow c_{I}^{-}$, and one can investigate its properties. This is straightforward within density-functional treatments but becomes problematic in simulations of the present system where the necessary $L_{z}$ becomes prohibitively large.

We should note that the shapes of the profiles for the 
density $c(z)$ and order parameters $s(z)$ and $\Delta(z)$ and their development with increasing $c_{b}$ are quite similar to those found for the Zwanzig model [21].

The subtleties of wetting are not important for the confined fluid where the separation of the (identical) walls is finite, and we find a first-order capillary nematization transition for a fluid confined by two parallel hard walls. The coexisting densities of the capillary isotropic (with biaxial nematic films at the walls) and condensed nematic phase were determined by Gibbs ensemble simulations. The difference in the coexisting densities decreased as the wall separation was reduced and no coexistence was found for reduced wall separation $h \equiv H /(L+D)<2.25$. Our results point to a capillary nematization transition ending in a capillary critical point at a critical (reduced) wall separation $2.25<h_{c}<2.375$. It is very pleasing that all the key features of the capillary nematization transition, even the estimate of the reduced critical wall separation $h_{c}$, are consistent with the theoretical predictions of the Zwanzig model [14,21], in which the orientations of the rods are restricted to three orthogonal directions. This attests to the universality of the phenomena we observe.

\section{ACKNOWLEDGMENTS}

R.v.R. and M.D. thank Bristol University for hospitality during the initial stages of this work, which was supported financially by the grants ERBFMBICT971869 of the TMR program and GR/L89013 of the EPSRC. We thank M.P. Allen for helpful discussions. This work is part of the research program of the "Stichting voor Fundamenteel Onderzoek der Materie (FOM),', which is financially supported by the "Nederlandse Organisatie voor Wetenschappelijk Onderzoek (NWO).',
[1] F. Reinitzer, Monatsch. Chem. 9, 421 (1888).

[2] H. Zocher, Z. Anorg. Chem. 147, 91 (1925).

[3] F.C. Bawden, N.W. Pirie, J.D. Bernal, and I. Fankuchen, Nature (London) 138, 1051 (1936).

[4] L. Onsager, Phys. Rev. 62, 558 (1942); Ann. (N.Y.) Acad. Sci. 51, 627 (1949).

[5] J.A.C. Veerman and D. Frenkel, Phys. Rev. A 41, 3237 (1990).

[6] P. Bolhuis and D. Frenkel, J. Chem. Phys. 106, 666 (1997).

[7] M. Schmidt and H. Löwen, Phys. Rev. E 55, 7228 (1997).

[8] A. Poniewierski and R. Holyst, Phys. Rev. A 38, 3721 (1988).

[9] A. Poniewierski, Phys. Rev. E 47, 3396 (1993).

[10] Z.Y. Chen and S.M. Cui, Phys. Rev. E 52, 3876 (1995).

[11] Y. Mao, P. Bladon, H.N.W. Lekkerkerker, and M.E. Cates, Mol. Phys. 92, 151 (1997).

[12] M.P. Allen, Mol. Phys. 96, 1391 (1999).

[13] M.P. Allen, J. Chem. Phys. 112, 5447 (2000).

[14] R. van Roij, M. Dijkstra, and R. Evans, Europhys. Lett. 49, 350 (2000).

[15] R. Evans, J. Phys.: Condens. Matter 2, 8989 (1990).

[16] J.M. Brader, M. Dijkstra, and R. Evans, Phys. Rev. E 63,
041405 (2001).

[17] R. Evans, Adv. Phys. 28, 143 (1979).

[18] J.E. Finn and P.A. Monson, Phys. Rev. A 39, 6402 (1989).

[19] Note that in Ref. [12], the two confining walls are impenetrable to the centers of the ellipsoids, not to the whole ellipsoid. Such a wall gives rise to homeotropic ordering, i.e., the director is perpendicular to the wall.

[20] D. Frenkel and B. Smit, Understanding Molecular Simulations: From Algorithms to Application (Academic Press, Cambridge, 1996).

[21] R. van Roij, M. Dijkstra, and R. Evans, J. Chem. Phys. 113, 7685 (2000).

[22] For completeness, we mention that the interfacial tension of the $I-N$ interface has recently been calculated by computer simulations for hard ellipsoids of revolution of elongation $e$ $=a / b=15$, where $a$ is the length of the semimajor axis and $b$ is the length of the two equal semiminor axes. The authors find that $\gamma_{\mathrm{IN}} b^{2} / k_{B} T=0.006 \pm 0.005$-see A.J. McDonald, M.P. Allen, and F. Schmid, Phys. Rev. E 63, 010701(R) (2001).

[23] A.Z. Panagiotopoulos, Mol. Phys. 62, 701 (1987). 